\title{
0 Tratamento Medicamentoso Habitual é Suficiente para Manter o Controle da Frequência Cardíaca nos Pacientes com Insuficiência Cardíaca?
}

\author{
Is Current Drug Therapy for Heart Failure Sufficient to Control Heart Rate of Patients? \\ Juliano Cardoso, ${ }^{1}$ Mateus Dressler de Espíndola, ${ }^{1}{ }^{10}$ Mauricio Cunha, ${ }^{1}$ Enock Netto, ${ }^{1}$ Cristina Cardoso, ${ }^{1}$ Milena \\ Novaes, ${ }^{1}$ Carlos Henrique Del Carlo, ${ }^{1}{ }^{\circledR}$ Euler Brancalhão, ${ }^{1}$ Alessandro Lyra Name, ${ }^{1}$ Antonio Carlos Pereira Barretto ${ }^{1}$ \\ Hospital Santa Marcelina - Cardiologia, ${ }^{1}$ São Paulo, SP - Brasil
}

\section{Resumo}

Fundamento: Estudos revelam que pacientes com insuficiência cardíaca (IC) e frequência cardíaca (FC) $<70$ batimentos por minuto (bpm) evoluem melhor e têm menor morbimortalidade em comparação com FC $>70$. Entretanto, muitos pacientes com IC mantêm FC elevada.

Objetivo: Avaliar se os pacientes acompanhados em ambulatório de cardiologia têm sua FC controlada e como estava a prescrição dos medicamentos que reduzem a mortalidade na IC.

Métodos: Foram analisados de forma consecutiva pacientes que passaram em consulta e que já acompanhavam em ambulatório de cardiologia, idade > 18 anos e com diagnóstico de IC e fração de ejeção do ventrículo esquerdo (FEVE) $<45 \%$. Os pacientes em ritmo sinusal foram divididos em dois grupos: FC $\leq 70$ bpm (G1) e FC $>70 \mathrm{bpm}$ (G2). Na análise estatística, foram utilizados os testes t de Student, Qui-quadrado. Foi considerado significante p $<0,05$. Utilizamos o programa Statistical Package for the Social Sciences (SPSS) para análise.

Resultados: Foram avaliados 212 pacientes de forma consecutiva. Destes, 41 (19,3\%) apresentavam fibrilação atrial ou eram portadores de marca-passo e foram excluídos desta análise; assim, 171 pacientes foram analisados. Os pacientes em ritmo sinusal tinham idade média de 63,80 anos $( \pm 11,77)$, sendo 59,6\% homens e FEVE média de $36,64 \%( \pm 7,79)$. Com relação à etiologia, a isquêmica estava presente em 102 pacientes $(59,65 \%)$, enquanto a cardiopatia chagásica em 17 pacientes (9,9\%); 131 pacientes eram hipertensos (76,6\%), enquanto 63 pacientes $(36,84 \%)$ eram diabéticos. Quanto à FC, 101 pacientes apresentaram FC $\leq 70 \mathrm{bpm}(59,06 \%)$ G1 e 70 pacientes (40,93\%) FC > 70 bpm (G2). A FC média no G1 foi de 61,53 bpm $( \pm 5,26)$ e no G2, 81,76 bpm $( \pm 9,52), p<0,001$. A quase totalidade dos pacientes $(98,8 \%)$ estava sendo tratada com carvedilol prescrito na dose média de 42,14 $\mathrm{mg} / \mathrm{dia}( \pm 18,55)$ no G1 versus $42,48 \mathrm{mg} / \mathrm{dia}( \pm 21,14)$ no $\mathrm{G} 2, \mathrm{p}=0,911$. A digoxina foi utilizada em $5,9 \% \mathrm{dos}$ pacientes no $\mathrm{G} 1$ versus $8,5 \%$ no $\mathrm{G} 2, \mathrm{p}=0,510$. A dose média de digoxina no G1 foi de $0,19 \mathrm{mg} / \mathrm{dia}( \pm 0,06)$ e no G2 foi de $0,19 \mathrm{mg} / \mathrm{dia}( \pm 0,06), p=0,999$. A maioria dos pacientes $(87,72 \%)$ utilizou o inibidor da enzima de conversão da angiotensina (IECA) ou bloqueador do receptor da angiotensina (BRA), e 56,72\% utilizaram espironolactona. A dose média de enalapril foi de $28,86 \mathrm{mg} / \mathrm{dia}( \pm 12,68)$ e de $B R A$ foi de $87,80 \mathrm{mg} / \mathrm{dia}( \pm 29,80)$. A maioria dos pacientes utilizou IECA ou BRA e com doses adequadas.

Conclusão: O estudo revelou que 40,93\% dos pacientes estavam com FC acima de 70 bpm, apesar de o betabloqueador ter sido prescrito para praticamente todos os pacientes e em doses elevadas. Outras medidas precisam ser adotadas para manter a FC mais controlada nesse grupo de frequência mais elevada. (Arq Bras Cardiol. 2020; 115(6):1063-1069)

Palavras-chave: Insuficiência Cardíaca; Frequência Cardíaca; Tratamento Farmacológico; Adesão à Medicação; Digoxicina; Morbidade e Mortalidade; Fibrilação Atrial; Marca-Passo.

Correspondência: Mateus Dressler de Espíndola •

Hospital Santa Marcelina - Cardiologia - Rua Santa Marcelina, 177. CEP 03614-000, Itaquera São Paulo, SP - Brasil

E-mail: mateusespindola@hotmail.com

Artigo recebido em 11/02/2019, revisado em 12/09/2019, aceito em 23/10/2019

DOI: https://doi.org/10.36660/abc.20190090 


\begin{abstract}
Background: Studies have shown that heart failure (HF) patients with heart rate (HR) $<70$ bpm have had a better clinical outcome and lower morbidity and mortality compared with those with HR $>70 \mathrm{bpm}$. However, many HF patients maintain an elevated HR.
\end{abstract}

Objective: To evaluate HR and the prescription of medications known to reduce mortality in HF patients attending an outpatient cardiology clinic.

Methods: We consecutively evaluated patients seen in an outpatient cardiology clinic, aged older than 18 years, with diagnosis of HF and left ventricular ejection fraction (LVEF) $<45 \%$. Patients with sinus rhythm were divided into two groups - HR $\leq 70$ bpm (G1) and HR $>70$ bpm (G2). The Student's t-test and the chi-square test were used in the statistical analysis, and a p-value < 0.05 was considered statistically significant. The SPSS software was used for the analyses.

Results: A total of 212 consecutive patients were studied; 41 (19.3\%) had atrial fibrillation or had a pacemaker implanted and were excluded from the analysis, yielding 171 patients. Mean age of patients was $63.80 \pm 11.77$ years, 59.6\% were men, and mean LVEF 36.64 \pm 7.79\%. The most prevalent HF etiology was ischemic ( $n=102 ; 59.6 \%)$, followed by Chagasic ( $n=17 ; 9.9 \%)$. One-hundred thirty-one patients (76.6\%) were hypertensive and 63 (36.8\%) diabetic. Regarding HR, 101 patients had a HR $\leq 70$ bpm (59.1\%) and 70 patients (40.93\%) had a HR > 70

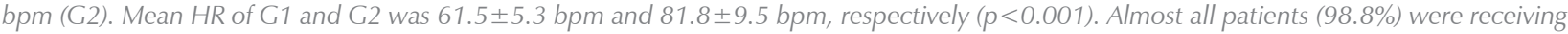
carvedilol, prescribed at a mean dose of $42.1 \pm 18.5 \mathrm{mg} /$ day in G1 and $42.5 \pm 21.1 \mathrm{mg} /$ day in G2 ( $p=0.911)$. Digoxin was used in 5.9\% of patients of G1 and 8.5\% of G2 ( $p=0.510)$. Mean dose of digoxin in G1 and G2 was 0.19 $\pm 0.1 \mathrm{mg} /$ day and $0.19 \pm 0.06 \mathrm{mg} /$ day, respectively $(p=0,999)$. Most patients (87.7\%) used angiotensin converting enzyme inhibitors (ACEI) or angiotensin II receptor blockers (ARB), and 56.7\% used spironolactone. Mean dose of enalapril was $28.9 \pm 12.7 \mathrm{mg} /$ day and mean dose of ARB was $87.8 \pm 29.8 \mathrm{mg} / \mathrm{day}$. The doses of ACEI and $A R B$ were adequate in most of patients.

Conclusion: The study revealed that HR of $40.9 \%$ of patients with HF was above 70 bpm, despite treatment with high doses of beta blockers. Further measures should be applied for HR control in HF patients who maintain an elevated rate despite adequate treatment with beta blocker. (Arq Bras Cardiol. 2020; 115(6):1063-1069)

Keywords: Heart Failure; Heart Rate; Drug Therapy; Medication Adherence; Digoxine; Morbidity \& Mortality; Atrial Fibrillation; Pacemaker, Artificial.

Full texts in English - http://www.arquivosonline.com.br

\section{Introdução}

A insuficiência cardíaca (IC) é uma síndrome cada vez mais frequente que evolui com elevada morbidade e mortalidade nas formas avançadas, sendo a fase final comum das cardiopatias. ${ }^{1}$

Apesar da gravidade da doença, o tratamento correto conforme as diretrizes melhora a qualidade de vida e reduz a mortalidade. ${ }^{2,3}$ Apesar das evidências da sua efetividade, a prescrição dos medicamentos que modificam o prognóstico é inferior ao desejado, como demonstrado em alguns registros recentes. ${ }^{4-6}$ Ao analisar os potenciais motivos para a subprescrição de fármacos direcionados aos pacientes com IC, é possível destacar hipotensão, idade avançada, além do receio de possíveis efeitos colaterais. ${ }^{6,7}$ Outro provável motivo para não alcançar as doses de eficácia comprovada pode ser a falta de metas objetivas no tratamento da IC, assim como fazemos no tratamento das dislipidemias e da hipertensão arterial. ${ }^{8,9}$ Talvez devêssemos perseguir no tratamento da IC algumas metas objetivas, como o controle mais rígido da frequência cardíaca (FC). A FC nos pacientes com IC tem se mostrado um guia importante para avaliação de eficácia do tratamento. Para os pacientes com IC, fração de ejeção do ventrículo esquerdo (FEVE) reduzida e em ritmo sinusal, o estudo SHIFT documentou que reduzir a FC para valores inferiores a 70 batimentos por minuto (bpm) modifica o prognóstico. Entretanto, uma parcela dos nossos pacientes ainda apresenta FC acima do desejado. ${ }^{10}$

Neste trabalho, procuramos verificar em um ambulatório de um grande hospital terciário da zona leste da cidade de São Paulo se os pacientes em ritmo sinusal apresentavam FC controlada ( $\mathrm{FC} \leq 70 \mathrm{bpm}$ ). Também avaliamos se a medicação para IC estava adequada conforme as diretrizes. ${ }^{2}$

\section{Material e Métodos}

Para realizar esta pesquisa avaliamos os pacientes com IC que foram atendidos consecutivamente no ambulatório de cardiologia no período de janeiro de 2016 a março de 2017 com FEVE $<45 \%$, em tratamento da IC há mais de 6 meses. Avaliamos dados demográficos, etiologia da cardiopatia, ritmo cardíaco, pressão arterial, FC e o tratamento medicamentoso, verificando as doses alcançadas com os diferentes fármacos.

Os critérios de inclusão foram: idade $>18$ anos, diagnóstico de IC, FEVE $<45 \%$ e ritmo sinusal. Para fim de análise, os pacientes foram divididos em dois grupos, um com $\mathrm{FC} \leq 70$ bpm (G1) e outro com FC > 70 bpm (G2).

Foi avaliada a prescrição dos três grupos de medicações que comprovadamente modificam a história natural da IC: vasodilatadores (inibidor da ECA [IECA] ou bloqueador dos receptores da angiotensina II [BRA] ou hidralazina e nitrato), espironolactona e betabloqueador; somente naqueles com disfunção renal e com hiperpotassemia persistente e que não toleravam pelo menos $50 \%$ de IECA/BRA, os vasodilatadores utilizados eram hidralazina e nitrato. Já nos pacientes em uso de dose plena de IECA/BRA, betabloqueador e espironolactona e que permaneciam sintomáticos, era adicionado ao tratamento o uso de hidralazina e nitrato.

A dose considerada correta para o IECA foi de $20 \mathrm{mg} \mathrm{2x/}$ dia de enalapril ou dose equivalente de captopril (150 mg/ dia). Para os BRA, a dose considerada correta foi de 100 a 150 $\mathrm{mg} /$ dia de losartana. Para espironolactona, a dose plena foi de $25 \mathrm{mg} /$ dia. Para os betabloqueadores, a dose considerada plena foi de $25 \mathrm{mg}$ 2x/dia para o carvedilol. ${ }^{2}$ Identificaramse também o percentual e a dose de prescrição de digoxina, hidroclorotiazida e furosemida, medicações frequentemente prescritas para os pacientes com IC. ${ }^{2}$ 
Para realização deste trabalho, houve aprovação do projeto pelo Comitê de Ética e Pesquisa da Casa de Saúde Santa Marcelina, sob parecer $n^{\circ} 13.10 .805$.

\section{Análise Estatística}

$\mathrm{Na}$ apresentação das características da população, as variáveis contínuas com distribuição normal são apresentadas como média \pm desvio padrão. As variáveis categóricas são apresentadas como número (porcentagem). O teste de Kolmogorov-Smirnov foi utilizado para testar a normalidade dos dados ( $p>0,05=$ distribuição normal). Na comparação dos grupos, as variáveis contínuas são apresentadas como média \pm desvio padrão. Foi utilizado o teste $t$-Student não pareado para as variáveis com distribuição normal. Os testes utilizados foram bicaudais e o valor de $p<0,05$ foi considerado como estatisticamente significante. Na comparação das características, foram utilizados os testes do Qui-quadrado ou Teste Exato de Fisher para as variáveis categóricas. Todas as análises estatísticas foram realizadas com o software estatístico Statistical Package for the Social Sciences (SPSS).

\section{Resultados}

Foram analisados 212 pacientes de forma consecutiva. Deste grupo, 41 (19,3\%) apresentavam fibrilação atrial ou eram portadores de marca-passo e foram excluídos desta análise. Portanto, analisamos 171 pacientes em ritmo sinusal. A idade média foi de 63,80 anos $\pm 11,77$, sendo $59,6 \%$ homens, FEVE média $36,64 \% \pm 7,79$ e Pro-BNP médio de $1663,95 \mathrm{pg} / \mathrm{mL} \pm 2158,77$. A etiologia isquêmica ocorreu em 102 pacientes (59,65\%). Diabetes estava presente em 63 pacientes (36,84\%), e 131 pacientes (76,6\%) apresentavam hipertensão arterial. Na Tabela 1, apresentamos as características clínicas dos pacientes e o tratamento que vinham recebendo. Na Tabela 2, apresentamos as doses dos medicamentos prescritos (mg/dia).

$\mathrm{Na}$ Tabela 3, estão demonstradas as diferenças entre os grupos de acordo com a frequência cardíaca.

\section{Discussão}

Nosso estudo revelou que 40,93\% dos pacientes avaliados apresentavam FC maior que 70 bpm, apesar de o carvedilol ter sido utilizado em mais de $98 \%$ dos pacientes e com dose média elevada, acima de $42 \mathrm{mg} /$ dia. Os dados deste estudo revelam que, na maioria dos casos, foi possível prescrever corretamente os medicamentos que modificam o prognóstico na IC. A frequência de prescrição foi mais elevada que as descritas nos registros internacionais, bem como no Registro Brasileiro BREATHE. ${ }^{4-6}$ Os pacientes estavam recebendo, em sua maioria, as doses-alvo dos medicamentos, como indicado pelas diretrizes. ${ }^{2,3}$

Esses resultados permitem supor que a não prescrição dos medicamentos pelos médicos, na maioria das vezes, decorre da falta de tentativa em aumentar as doses. É importante ressaltar que a não prescrição em doses, de pelo menos $50 \%$ da dose-alvo dos medicamentos, implica menor proteção aos pacientes e um risco maior de morte e hospitalizações. ${ }^{6,7}$
Tabela 1 - Características clínicas e tratamento dos pacientes em ritmo sinusal estudados.

\begin{tabular}{|c|c|}
\hline Pacientes n & 171 \\
\hline Idade em anos & $63,80 \pm 11,77$ \\
\hline Isquêmica & $102(59,65 \%)$ \\
\hline Chagásica & $17(9,9 \%)$ \\
\hline Idiopática & $29(17 \%)$ \\
\hline \multicolumn{2}{|l|}{ Comorbidades $n$} \\
\hline Diabetes & $63(36,84 \%)$ \\
\hline Hipertensão arterial & $131(76,6 \%)$ \\
\hline \multicolumn{2}{|l|}{ Dados clínicos } \\
\hline PAS em mmHg & $119,56 \pm 18,69$ \\
\hline FC em bpm & $69,81 \pm 12,34$ \\
\hline NT-proBNP & $1663,95 \pm 2158,77$ \\
\hline \multicolumn{2}{|c|}{ Dados do ecocardiograma } \\
\hline DDVE em mm & $61,34 \pm 7,79$ \\
\hline DSVE em mm & $50,33 \pm 8,25$ \\
\hline FEVE percentual & $36,64 \pm 6,73$ \\
\hline \multicolumn{2}{|c|}{ Medicamentos prescritos $\mathrm{n}$} \\
\hline Furosemida & $90(52,63 \%)$ \\
\hline Hidroclorotiazida & $25(14,61 \%)$ \\
\hline IECA/BRA & $150(87,72 \%)$ \\
\hline Betabloqueador & $169(98,83 \%)$ \\
\hline Espironolactona & $97(56,72 \%)$ \\
\hline Hidralazina & $30(17,54 \%)$ \\
\hline Nitrato & $42(24,56 \%)$ \\
\hline Digoxina & $12(7,01 \%)$ \\
\hline
\end{tabular}

PAS: pressão arterial sistólica; FC: frequência cardiaca; DDVE: diâmetro diastólico do ventrículo esquerdo; DSVE: diâmetro sistólico do ventrículo esquerdo; FEVE: fração de ejeção do ventrículo esquerdo; NT-proBNP. porção N terminal do peptídio natriurético do tipo "B"; IECA: inibidor da enzima conversora da angiotensina; BRA: bloqueador do receptor da angiotensina.

Nossos dados diferem dos dados de Registros como o BIOSTAT-HF, no qual somente $60 \%$ dos pacientes estavam recebendo dose de $50 \%$ ou mais da dose-alvo de IECA ou BRA, e cerca de $40 \%$ recebiam dose de $50 \%$ ou mais da dosealvo de betabloqueador, doses estas associadas à redução de mortalidade, como bem demonstrado nesse Registro. ${ }^{6}$ O mesmo se aplica para dados brasileiros quando o Registro BREATHE mostrou que 83,4\% estavam recebendo IECA/BRA na alta do hospital e $63,1 \%$, um betabloqueador. ${ }^{5}$ No Registro QUALIFY, apesar de a maioria dos pacientes estar recebendo prescrição de IECA/BRA $(87,5 \%)$ ou betabloqueador $(87 \%)$, somente $14,8 \%$ recebiam a dose-alvo e $51,8 \%$ recebiam dose superior a $50 \%$ da dose-alvo de IECA. Ao passo que, nesse mesmo estudo, 27,9\% estavam recebendo dose-alvo de betabloqueador e $51,8 \%$ receberam dose $50 \%$ ou mais da dose-alvo do betabloqueador. ${ }^{4}$ Em nossos pacientes, $79,09 \%$ estão recebendo dose-alvo de IECA, sendo que 53,63\% destes estavam recebendo $40 \mathrm{mg} /$ dia de enalapril, e 58,47\% 


\begin{tabular}{lc}
\hline $\begin{array}{l}\text { Tabela } 2 \text { - Dose média dos medicamentos prescritos (mg/dia) e } \\
\text { desvio padrão }\end{array}$ & Dose \\
\hline Medicamento & $52,31 \pm 26,15$ \\
\hline Furosemida & $26,09 \pm 5,10$ \\
\hline Hidroclotiazida & $28,86 \pm 12,68$ \\
\hline Enalapril & $87,80 \pm 29,80$ \\
\hline Losartana & $42,28 \pm 19,65$ \\
\hline Carvedilol & $25,00 \pm 4,77$ \\
\hline Espironolactona & $96,55 \pm 59,35$ \\
\hline Hidralazina & $53,90 \pm 15,60$ \\
\hline Isossorbida & $0,19 \pm 0,06$ \\
\hline Digoxina &
\end{tabular}

recebiam dose-alvo de betabloqueador, sendo que 15\% destes, com doses superiores a $50 \mathrm{mg} /$ dia do carvedilol. Uma dose de $50 \%$ ou mais da dose-alvo de IECA foi prescrita para $97,27 \%$ dos pacientes e $88,88 \%$ recebiam dose de $50 \%$ ou mais de betabloqueador (carvedilol) (Figura 1).

Nosso estudo revelou também que mesmo bem tratados com dose média de carvedilol de 42,48 mg/dia, muitos pacientes ainda apresentam FC acima de 70 bpm; 40\% em ritmo sinusal apresentavam FC $>70$ bpm (Figura 2). Esses resultados estão de acordo com os da literatura, pois a totalidade dos artigos que aborda FC em pacientes em tratamento da IC com IECA, betabloqueador e espironolactona descreve que percentual acentuado de pacientes continua com FC acima de 70 bpm, apesar do tratamento. Vale ressaltar que, em muitos desses artigos, a dose de betabloqueador não é elevada, com a maioria permanece com dose abaixo de $50 \%$ da dose-alvo. ${ }^{6,7}$

Tabela 3 - Dados clínicos e de tratamento dos pacientes em ritmo sinusal de acordo com FC

\begin{tabular}{|c|c|c|c|}
\hline & G1 (FC $\leq 70 \mathrm{bpm})$ & $\mathrm{G} 2 \mathrm{FC}>70 \mathrm{bpm}$ & p \\
\hline Pacientes & $101(59,06 \%)$ & $70(40,93 \%)$ & \\
\hline Homens & $62(61,38 \%)$ & $40(57,97 \%)$ & \\
\hline \multicolumn{4}{|c|}{ Etiologia da cardiopatia } \\
\hline Isquêmica & $59(58,41 \%)$ & $43(61,64 \%)$ & 0,938 \\
\hline Chagásica & $16(15,84 \%)$ & $3(4,28 \%)$ & 0,009 \\
\hline Não isquêmica & $26(25,74 \%)$ & $24(34,28 \%)$ & \\
\hline \multicolumn{4}{|l|}{ Comorbidades } \\
\hline Diabetes & $31(30,69 \%)$ & $32(45,71 \%)$ & 0,045 \\
\hline Hipertensão arterial & $82(81,18 \%)$ & $50(71,42 \%)$ & 0,108 \\
\hline \multicolumn{4}{|l|}{ Dados clínicos } \\
\hline PAS em mmHg & $119,76 \pm 17,87$ & $119,29 \pm 19,81$ & 0,871 \\
\hline FC em bpm & $61,53 \pm 5,26$ & $81,76 \pm 9,52$ & $<0,001$ \\
\hline NT-proBNP & $1625,09 \pm 2258,42$ & $1721,80 \pm 1999,91$ & 0,822 \\
\hline \multicolumn{4}{|c|}{ Dados do Ecocardiograma } \\
\hline DDVE em mm & $61,26 \pm 7,78$ & $61,46 \pm 7,82$ & 0,868 \\
\hline DSVE em mm & $49,84 \pm 8,42$ & $51,12 \pm 7,92$ & 0,356 \\
\hline FEVE em \% & $37,46 \pm 6,58$ & $35,46 \pm 6,78$ & 0,056 \\
\hline \multicolumn{4}{|c|}{ Dose dos medicamentos prescritos } \\
\hline Furosemida & $50,57 \pm 25,06$ & $54,74 \pm 27,41$ & 0,458 \\
\hline Hidroclorotiazida & $26,92 \pm 6,66$ & $25,00 \pm 0,00$ & 0,392 \\
\hline Enalapril & $29,77 \pm 12,38$ & $27,50 \pm 12,99$ & 0,361 \\
\hline Losartana & $80,43 \pm 33,75$ & $97,22 \pm 20,22$ & 0,076 \\
\hline Carvedilol & $42,14 \pm 18,55$ & $42,48 \pm 21,14$ & 0,911 \\
\hline Espironolactona & $24,79 \pm 4,84$ & $25,35 \pm 4,64$ & 0,585 \\
\hline Hidralazina & $111,11 \pm 67,81$ & $72,73 \pm 29,11$ & 0,097 \\
\hline Isossorbida & $55,77 \pm 16,21$ & $50,67 \pm 13,89$ & 0,325 \\
\hline Digoxina & $0,19 \pm 0,06$ & $0,19 \pm 0,06$ & 0,999 \\
\hline
\end{tabular}

PAS: pressão arterial sistólica; FC: frequência cardíaca; DDVE: diâmetro diastólico do ventrículo esquerdo; DSVE: diâmetro sistólico do ventrículo esquerdo; FEVE: fração de ejeção do ventrículo esquerdo; NT-proBNP: porção N terminal do peptídeo natriurético do tipo "B". 


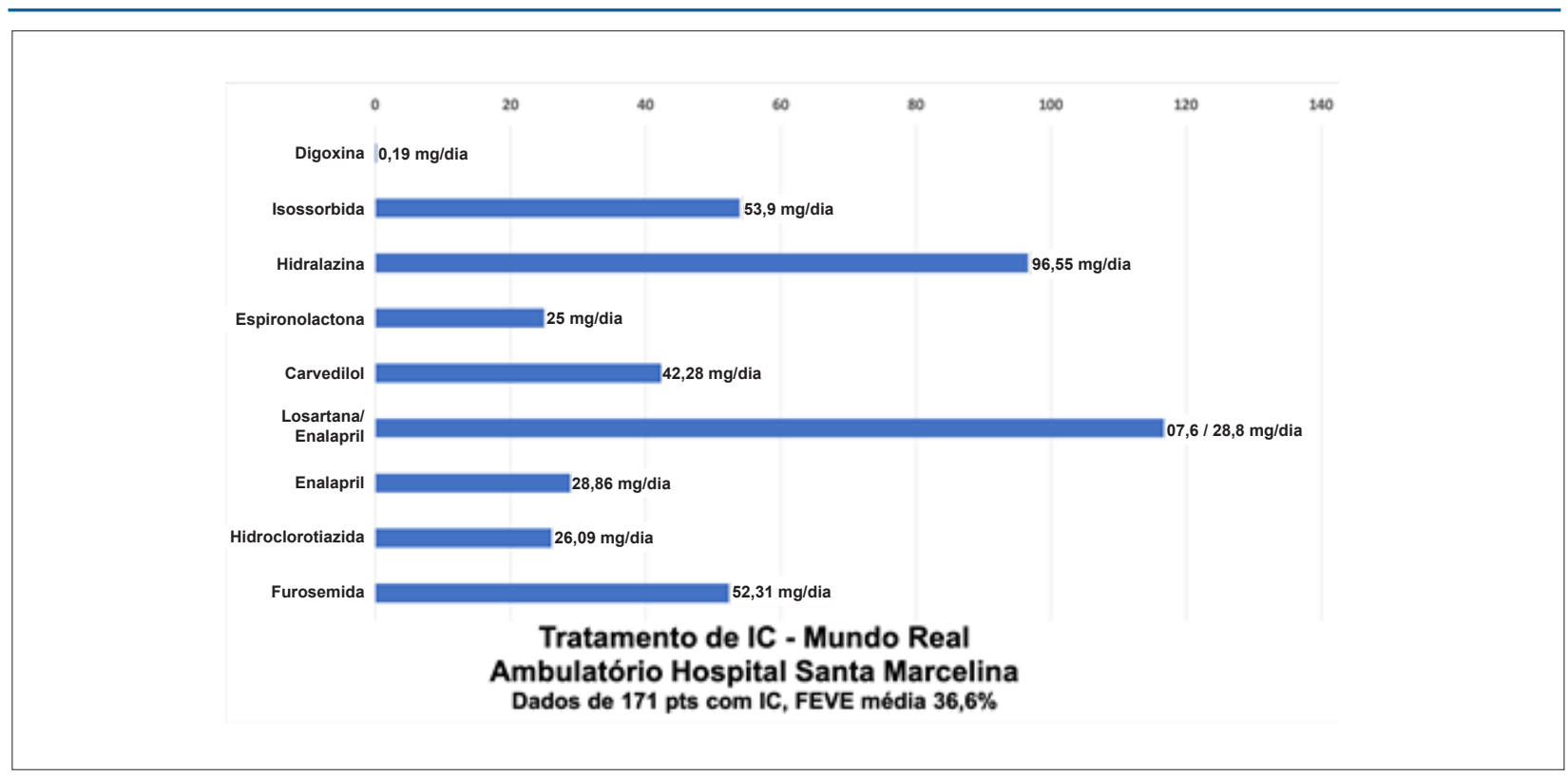

Figura 1 - Gráfico mostrando o percentual de pacientes tomando cada medicamento (\%) e a dose média prescrita para cada fármaco.

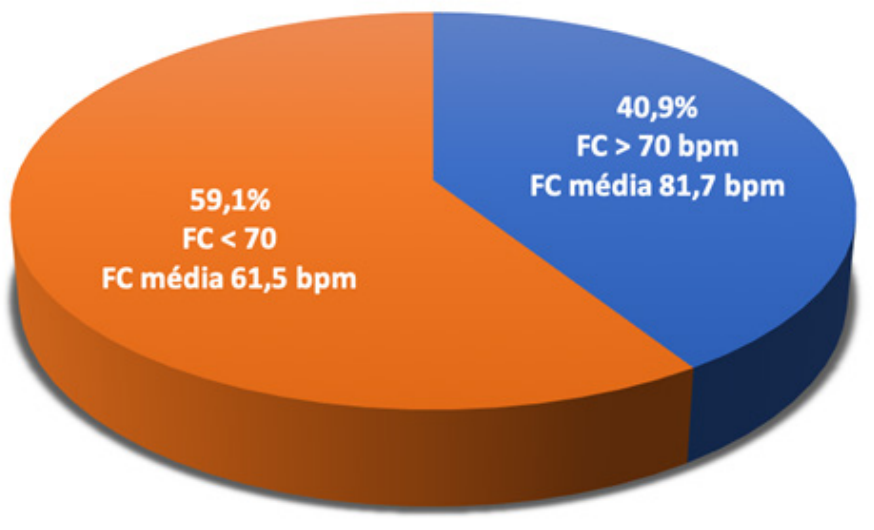

\section{Distribuição da FC em pacientes em ritmo sinusal}

Figura 2 - Percentual de pacientes em ritmo sinusal que apresentam frequência cardíaca (FC) maior ou menor que 70 bpm.

No Registro OPTIMIZE-HF, que avaliou 10.697 pacientes de hospitais dos EUA, constatou-se que a FC média na alta era de 76 bpm, e que não houve uma correlação com a dose do betabloqueador. Os pacientes com dose inferior a 25\% da dose-alvo de betabloqueador apresentaram FC média de 78 bpm, já os com a dose-alvo de 72 bpm, a FC elevada guardou relação com o prognóstico, sendo maior a morbimortalidade para aqueles com FC acima de 70 bpm. ${ }^{11}$ Na Duke University, verificou-se também que a maioria dos pacientes (73\%) tratados apresentava FC acima de $70 \mathrm{bpm}$. Tais pacientes com FC acima de 70 bpm apresentaram maior morbimortalidade
(RR 1,59), e foi observado que a FC elevada foi associada a maior custo de tratamento. ${ }^{12}$ Habal et al. ${ }^{13}$ verificou que o risco de morte dos pacientes era 59\% maior se os pacientes apresentassem FC acima de 90 bpm em relação aos com FC entre 61 a 70 bpm. ${ }^{13} \mathrm{O}$ estudo ASCEND-HF revelou que muitos pacientes persistiam com FC elevada, sendo que $85 \%$ dos pacientes apresentavam FC acima de 70 bpm, e esta FC elevada foi associada à maior mortalidade. ${ }^{14}$

No nosso estudo, embora expressivo o número de casos com FC $>70$ bpm, ela foi menor que a descrita nesses estudos 
comentados, possivelmente em decorrência do tratamento com doses mais elevadas dos betabloqueadores. Tal fato também foi observado em estudo com pacientes de consultório que estavam recebendo dose próxima à dose-alvo de carvedilol, no qual encontramos cerca de $35 \%$ dos pacientes com FC acima de 70 bpm. ${ }^{15}$

Um tema em discussão na literatura é o que seria mais importante para determinar uma boa evolução dos portadores de IC: se a dose-alvo do betabloqueador ou a redução da FC. Vale ressaltar que a redução da $\mathrm{FC}$ com os betabloqueadores não é igual para todos os pacientes. No estudo MERIT-HF, identificaramse dois grupos: um que teve redução da FC com doses baixas de metoprolol (dose média $76 \mathrm{mg} / \mathrm{dia}$ ) e outro que necessitou da dose-alvo para reduzir a FC (dose média $195 \mathrm{mg} / \mathrm{dia}$ ). ${ }^{16}$ Essa possível diferença pode ser geneticamente determinada, ${ }^{16}$ sendo alguns pacientes muito responsivos às doses dos medicamentos. ${ }^{16}$ Quanto aos resultados do estudo, os autores sinalizaram que a redução de eventos não foi estatisticamente diferente nos dois grupos, indicando que a redução de FC foi mais importante que a dose do betabloqueador na redução de eventos cardiovasculares. ${ }^{16}$

Considerando-se a redução da FC, é importante ressaltar que o estudo SHIFT indicou que a FC-alvo seria inferior a 70 bpm. ${ }^{10}$ No próprio estudo SHIFT, também se documentou que a redução da FC foi mais importante que a dose do betabloqueador na promoção da redução de eventos cardiovasculares. ${ }^{17}$

Quando se aborda a FC, é importante ressaltar que a maior redução de eventos tem sido descrita quando se atinge FC inferior a 64 bpm, como bem demonstrado nos estudos CHARM e CIBIS-ELD. ${ }^{18,19}$

Em metanálise com vários ensaios clínicos com betabloqueador, a sua prescrição foi associada à redução de mortalidade de 34\%, e observou-se que a redução da FC se correlacionou melhor com a redução de eventos do que com a dose do betabloqueador. ${ }^{20}$ Nesta metanálise para a redução de cada 5 bpm, observou-se redução de $18 \%$ no risco de morte e, quanto à dose, ela não foi determinante da redução de eventos, constatando-se redução de mortalidade de $26 \%$ para dose mais elevada e de $22 \%$ para as doses mais baixas. ${ }^{20}$

Nos estudos BIOSTAT-HF e na análise retrospectiva dos dados do estudo ACTION-HF, observou-se maior redução de eventos nos pacientes tratados com dose mais elevada do betabloqueador. ${ }^{6,21}$ No estudo BIOSTAT-HF, doses superiores a $50 \%$ da dose-alvo foram associadas à maior redução de mortalidade entre os 2.516 pacientes estudados. Os autores, na discussão, comentam que não conseguiram detectar diferença na evolução entre os tratados com mais de $50 \%$ da dose-alvo e aqueles tratados com dose alvo, mas doses mais baixas não protegeram os pacientes. ${ }^{6}$ No estudo ACTION-HF, os pacientes que tiveram a melhor evolução (maior redução de mortalidade) foram aqueles que tiveram redução da FC abaixo de 70 bpm com dose de $50 \%$ ou mais do betabloqueador. Os pacientes com dose mais baixa tiveram maior mortalidade. Quando se analisa somente os pacientes com dose mais baixa, aqueles que apresentavam FC menor que 70 bpm tiveram melhor evolução do que aqueles com FC acima de 70 bpm. ${ }^{21}$

Podemos concluir que os dois pontos são importantes, doses baixas de betabloqueador e FC acima de 70 bpm estão associadas a pior prognóstico. Os dados da literatura destacam a importância de avaliar a FC em todos os pacientes e, naqueles com FC acima de 70 bpm, otimizar o tratamento, quer aumentando a dose do betabloqueador se estiver sendo prescrita em dose baixa quer prescrevendo ivabradina para que seja possível reduzi-la, uma vez que a FC acima de 70 bpm vem se mostrando um excelente e fácil marcador de pior evolução. Vale lembrar que quanto mais elevada a FC, pior o prognóstico. Devemos nos policiar para evitar a inércia de deixar para a próxima avaliação a mudança de conduta quando encontramos um paciente em ritmo sinusal com FC acima de 70 bpm. É importante também lembrar que doses baixas de betabloqueador não tiveram sua eficácia comprovada. Além disso, nos pacientes que apresentam FC elevada apesar do uso da dose correta de betabloqueador, podemos utilizar a ivabradina, que é um inibidor dos canais $f$ e que reduz a FC nos pacientes em ritmo sinusal. ${ }^{2}$

Tivemos como limitação deste estudo o fato de ser unicêntrico, tendo, portanto, as limitações relacionadas a esse fato. $\mathrm{O}$ estudo teve como ponto forte revelar que, dentre os pacientes estudados, apesar de serem bem tratados do ponto de vista da IC, inclusive com doses adequadas de betabloqueador, muitos ainda permanecem com FC mais elevada. ${ }^{22}$ Fato que relevante, pois está relacionado com o prognóstico desses pacientes

\section{Conclusão}

Dos pacientes avaliados em ritmo sinusal, 40,93\% ainda apresentavam FC acima de $70 \mathrm{bpm}$, apesar de o betabloqueador ter sido prescrito para $98,83 \%$ dos pacientes, e com dose média elevada $(42,28 \mathrm{mg} / \mathrm{dia} \pm 19,65)$. Outras medidas precisam ser adotadas para manter a FC mais controlada nesse grupo que persiste com a FC elevada. Os vasodilatadores foram utilizados com frequência e com dose média adequada.

\section{Contribuição dos autores}

Concepção e desenho da pesquisa: Cardoso J, Cardoso C, Novaes M, Barretto ACP; Obtenção de dados: Cunha M, Netto E, Novaes M, Del Carlo CH, Brancalhão E; Análise e interpretação dos dados: Cardoso J, Brancalhão E; Análise estatística: Brancalhão E; Redação do manuscrito: Cardoso J; Revisão crítica do manuscrito quanto ao conteúdo intelectual importante: Cardoso J, Espíndola MD, Cardoso C, Name AL, Barretto ACP.

\section{Potencial conflito de interesses}

Declaro não haver conflito de interesses pertinentes.

\section{Fontes de financiamento}

O presente estudo não teve fontes de financiamento externas.

\section{Vinculação acadêmica}

Não há vinculação deste estudo a programas de pósgraduação. 


\section{Referências}

1. Brasil. Ministério da Saúde. Datasus: mortalidade - 1996 a 2015, pela CID10. Brasília, 2017.

2. Brasil.Ministério da Saúde Datasus. [Citado em 2018 abril 10]. [Disponível em: http://tabnet.datasus.gov.br/cgi/deftohtm.exe?sim/cnv/obt10uf.de.

3. Comitê Coordenador da Diretriz Brasileira de Insuficiência Cardíaca Crônica e Aguda. Arq Bras Cardiol. 2018; 111(3):436-539.

4. Ponikowski P, Voors AA, Anker SD, Bueno H, Cleland JGF, Coats AJS, et al. 2016 ESC Guidelines for the diagnosis and treatment of acute and chronic heart failure. The task force for the diagnosis and treatment of acute and chronic heart failure of the European Society of Cardiology. Eur Heart J. 2016;18(8):891-975.

5. Komadja M, Cowie MR, Tavazi L, Ponikowski P, Anker SD, Filippatos GS, et al. on behalf of the QUALIFY investigators. Physicians guideline adherence is associated with better prognosis in outpatients with heart failure and reduced ejection fraction: the QUALIFY international registry. Eur J Heart Fail. 2017;19(11):1414-23

6. Albuquerque DC, Souza Neto JD, Bacal F, Rhode LE, Bernardez-Pereira S Berwanger O, et al. Investigadores Estudo BREATHE. I Registro Brasileiro de Insuficiência Cardíaca aspectos clínicos, qualidade assistencial e desfechos hospitalares. Arq Bras Cardiol. 2015; 104(6):433-42.

7. Ouwerkerk W, Voors AA, Anker SD, Cleland JG, Filippatos G, Van der Harst $\mathrm{P}$, et al. Determinants and clinical outcome of uptitration of ACE-Inhibitors and beta-blockers in patients with heart failure: a prospective European study. Eur Heart J. 2017;38(24):1883-90.

8. Komajda M, Anker SD, Cowie MR, Filippatos GS, Mengele B, Ponikowski $\mathrm{P}$, et al. Physicians' adherence to guideline recommended medications in heart failure with reduced ejection fraction: data from the QUALIFY global survey. Eur J Heart Fail. 2016; 18(5):514-22.

9. Faludi AA, Izar MCO, Saraiva JFK, Chacra APM, Bianco HT, Afiune Neto J, et al. Atualização da Diretriz Brasileira de Dislipidemias e Prevenção da aterosclerose - 2017. Arq Bras Cardiol. 2017;109(2 supl 1):1-76.

10. Malachias MVB, Barbosa ECD, Martim JF, Rosito GBA, Toledo JY Passarelli O Jr, et al. 7a Diretriz Brasileira de Hipertensão Arterial. Arq Bras Cardiol. 2016; 107(3 supl 3):1-83.

11. Swedberg K, Komajda M, Bohm M, Borer JS, Ford L, Dubost-Brama A, et al. for the SHIFT investigators. Ivabradine and outcomes in chronic heart failure (SHIFT): a randomized placebo-controlled study. Lancet. 2010; 376(9744):875-85.

12. De Voore AD, Xiaojuan Mi,, Mentz RJ,Fonarow GC, Van Dyke M, Maya J, Hardy C, et al.Discharge heart rate and betablocker dose in patients hospitalized with heart failure: Findings from OPTIMIZE -HF registry. Am Heart J. 2016;173:172-8.

13. De Vore AD, Schulte PJ, Mentz RJ, Hardy NC, Kelly JP, Velazquez EJ, et al Relation of elevated heart rate in patients with heart failure with reduced ejection fraction to one year outcome and costs. Am J Cardiol. 2016; 117(6):946-51.

14. Habal MV, Liu PP, Austin PC, RossHI, Newton GE, Wang X, et al. Association of Heart Rate at Hospital Discharge with Mortality and Hospitalizations in Patients with Heart Failure. Circ Heart Fail. 2014;7(1):12-20.

15. Kitai T, Grodin J, Mentz RJ, Hernandez AF, Butler J, Metra M, et al. Insufficient reduction in heart rate during hospitalization despite beta-blocker treatment in acute decompensated heart failure: insights from the ASCEND-HF trial. Eur J Heart Fail. 2017;19(2):241-9.

16. Moreno IB, Del Carlos CH, Pereira-Barretto AC. Tratamento otimizado e reduçõ da frequência cardáiaca na insuficiência cardíaca crônica. Arq Bras Cardiol. 2013; 101(5):442-8.

17. Wikstrand J, Hjalmarson A, Waagstein F, et al. for the MERIT-HF study group. Dose of metoprolol CR/XL and clinical outcomes in patients with heart failure. Analysis of the experience in Metoprolol CR/XL randomized intervention trial in chronic heart failure (MERIT-HF). J Am Coll Cardiol. 2002; 40(3):491-8

18. Swedberg K, Komajda M, Bohm M, Borer J, Robertson M, Tavazzi L, et al Effects on outcomes of heart rate reduction by ivabradine in patients with congestive heart failure: Is there an influence of beta-blocker dose: J Am Coll Cardiol. 2012; 59(22):1938-45.

19. Castagno D, Skali H, Takeuchi M, Swedberg K, Yusuf S, Granger CB, et al Association of heart rate and outcomes in a broad spectrum of patients with chronic heart failure. Results from the CHARM program. J Am Coll Cardiol. 2012; 59(20):1785-95

20. Dungen HD, Musial-Bright L, Inkrot S, Apostolovic S, Edelmann f, Lainscak $M$, et al. Heart rate following short-term beta-blocker titration predicts all-cause mortality in elderly chronic heart failure patients: insight from the CIBIS-ELD trial. Eur J Heart Fail. 2014;16(8):907-14.

21. McAlister FA; Wiebe N, Ezekowitz JA, Leung AA, Armstrong PW. Metaanalysis: Beta-Blocker Dose, Heart Rate Reduction, and Death in Patients With Heart Failure. Ann Intern Med. 2009;150(11):784-94.

22. Fiuzat M, Daniel Wojdyla D, Pina I, Kitzman D, Flkeg J, Keteyian SJ,et al. Heart Rate or Beta-Blocker Dose? Association With Outcomes in Ambulatory Heart Failure Patients With Systolic Dysfunction Results From the HF-ACTION Trial. J Am Coll Cardiol Heart Fail. 2016;4(2):109-15. 